\title{
Stability and Thermodynamics Properties of CrFeNiCoMn/Pd High Entropy Alloys from First Principles
}

\author{
Nguyen-Dung $\operatorname{Tran}^{1} \cdot$ Arkapol Saengdeejing $^{1} \cdot \operatorname{Ken~Suzuki}^{1} \cdot$ Hideo Miura $^{1} \cdot$ \\ Ying Chen ${ }^{1}$
}

Submitted: 24 March 2021 / in revised form: 10 May 2021 / Accepted: 24 May 2021 / Published online: 28 June 2021

(c) ASM International 2021

\begin{abstract}
In this study, we focus on structural and thermodynamics properties, as well as phase stability of quinary $\mathrm{CrFeCoNiMn}$ and $\mathrm{CrFeCoNiPd}$ high-entropy alloys (HEA) for both equiatomic and non-equiatomic compositions. CrFeCoNiMn (Cantor alloy) is a widely studied fec alloy, while $\mathrm{CrFeCoNiPd}$ is a newly reported fcc alloy, being synthesized by intentionally substituting $\mathrm{Mn}$ in Cantor alloy by Pd which has a markedly different atomic size and electronegativity from the other constituent elements and has been achieved the better mechanical properties than Cantor alloy in experiments. DFT-based integrated approaches are conducted on these two quinary systems to calculate the structural, electronic structure and magnetic properties at zero $\mathrm{K}$, as well as the free energies as function of temperature including vibrational, configurational mixing entropy and thermal electronic effects. Various SQS models with about 200 atoms were created to simulate the equiatomic HEA and a special non-equiatomic HEA where a principal element has a rather high concentration while other four kinds of element have equal lower concentrations. Comparison between Mn- and Pd-HEAs in
\end{abstract}

This article is part of a special topical focus in the Journal of Phase Equilibria and Diffusion on the Thermodynamics and Kinetics of

High-Entropy Alloys. This issue was organized by Dr. Michael Gao, National Energy Technology Laboratory; Dr. Ursula Kattner, NIST; Prof. Raymundo Arroyave, Texas A\&M University; and the late Dr. John Morral, The Ohio State University.

\footnotetext{
Nguyen-Dung Tran

tran.nguyen.dung@rift.mech.tohoku.ac.jp

Ying Chen

ying@ rift.mech.tohoku.ac.jp

1 Fracture and Reliability Research Institute, School of Engineering, Tohoku University, 6-6-01 Aramakiaoba, Aobaku, Sendai 980-8579, Japan
}

both equiatomic and non-equiatomic compositions shows that the stability of $\mathrm{Mn}$ - and Pd-HEAs at zero $\mathrm{K}$ and finite temperature are dominated by different mechanisms, this can explain the recent experimental observation that a pronounced spatial fluctuation in atomic fraction is much wider in Pd-HEA rather than in Mn-HEA.

Keywords HEA - CrFeNiCoMn/Pd HEAs . Thermodynamics $\cdot$ DFT

\section{Introduction}

High-entropy alloys (HEAs) have been attracting a great of interest because of their unconventional mixing of multiple principal elements (at least 4 or 5), resulting in attractive mechanical, magnetic and electronic, chemical, as well as other physical properties. ${ }^{[1-5]}$ The novel properties of HEAs have been shown comparable and sometimes superior to those of commercial and traditional metallic alloys. Also, the ability of tailoring those properties of HEAs by controlling their compositions or modifying their microstructures makes HEAs future candidates for material design and applications. Much works have been undertaken in order to realize the potential of HEAs in various fields such as superalloy, irradiation and corrosion resistances, superconductor, catalyst, etc. ${ }^{[4,6-8]}$

Among hundreds of different HEA systems synthesized and investigated, the $\mathrm{CrFeNiCoMn}$, formed from five $3 \mathrm{~d}$ transition metal elements, so called Cantor alloy is one of the most interest. Objectives such as phase stability, local lattice distortion, microstructure, magnetic and electronic structure properties, and their effects on mechanical, elastic properties and others have been continuously studied and 
actively discussed. ${ }^{[4,5,9]}$ Experimental characterization has demonstrated the Cantor alloy having a single fec random solid solution phase at high temperature with remarkable combination of strength and ductility. ${ }^{[10-12]}$ Efforts to further enhance its mechanical properties are under way, focusing on modifying its compositions away from equiatomic center ${ }^{[13,14]}$ or by interstitial alloying. ${ }^{[15,16]}$ Few studies concentrated on interdiffusion in $\mathrm{CrFeNiCoMn}$ alloy using classical theoretical model or high-throughput technique. ${ }^{[17,18]}$ Yet for better understanding CrFeNiCoMn alloy and building up a sufficient thermodynamic database which would allow its potential applications being more realistic, more investigations are required both experimentally and theoretically.

Recently, Ding et al. have reported a new fcc equiatomic $\mathrm{CrFeNiCoPd}$ alloy, which is synthesized by intentionally substituting $\mathrm{Mn}$ in Cantor alloy with the $4 \mathrm{~d}$ element $\mathrm{Pd}$ which has a markedly different atomic size and electronegativity from the other constituent elements, showing that its strength is about 2.5 times higher than that of $\mathrm{CrFeNiCoMn}$ at similar grain size, and is comparable to that of advanced high-strength steels. ${ }^{[19]}$ Quite interestingly, the authors had noticed a pronounced difference in the distribution of constituent elements between $\mathrm{CrFeNi}$ $\mathrm{CoMn}$ and $\mathrm{CrFeNiCoPd}$ alloys: it is relatively random and uniform for the former, while it shows a great inhomogeneity and aggregation for the latter. Soon thereafter, Yin and Curtin have proposed a parameter-free model for random alloys which requires only solute misfit volumes, alloy elastic constants, and alloy lattice constant, capable of predicting $\mathrm{CrFeNiCoPd}$ strength in good agreement with the Ding et al.'s experimental finding. ${ }^{[20]}$ The model however cannot account for the different behaviors in the fluctuation in element distribution between $\mathrm{CrFeNiCoMn}$ and $\mathrm{CrFeNiCoPd}$ alloys. To this end, a closer look at atomistic, thermodynamic and energetic level for both equiatomic and non-equiatomic quinary $\mathrm{CrFeNiCoPd}$ alloys, as well as a detailed comparison with $\mathrm{CrFeNiCoMn}$ counterparts in a systematic manner are necessary. There are still only a few studies on CrFeNiCoPd HEAs compared to those on the Cantor alloy; and it is often that already investigated non-equiatomic compositions are not the same. Thus, a direct comparison between the two HEAs is somehow limited.

This work aims to reveal the similarities and differences between $\mathrm{CrFeNiCoMn}$ and $\mathrm{CrFeNiCoPd}$ HEAs in terms of structural, electronic structure and magnetic properties, as well as thermodynamics and energetics, and to investigate the Pd-sub-Mn substitution effect using the DFT-based integrated approaches. The usage of supercell approach including two equiatomic and two extreme non-equiatomic special quasirandom structures (SQSs) employed in the same manner for both $\mathrm{CrFeNiCoMn}$ and $\mathrm{CrFeNiCoPd}$ systems allows us to compare the two directly and systematically. Furthermore, two stability types identified in this work for $\mathrm{CrFeNiCoMn}$ and $\mathrm{CrFeNiCoPd} \mathrm{HEAs,} \mathrm{going}$ from equiatomic center toward non-equiatomic, gave an explanation to the Ding's experimental observation.

\section{Models and Computational Methods}

\subsection{Models}

Models of single phase solid-solution quinary HEAs were represented by Special Quasirandom Structures (SQSs) ${ }^{[23]}$ as shown in insets in Fig. 1. All these four equiatomic and non-equiatomic, fcc and bcc, SQS structures consist of the same number of atoms in supercell, 160, which is bigger than those used in Refs ${ }^{[24,25]}$ that claimed to be sufficient for modelling quinary HEA. The supercell lattice parameters of the starting fcc and bcc SQS structures, $a^{*}(1, b / a, c /$ a) and $(\alpha, \beta, \gamma)$ (in degree), are $\mathrm{a}^{*}(1,1.00,1.00) \&(90$, $106.3,108.7)$ and $\mathrm{a}^{*}(1,1.00,1.00) \&(93.8,93.8,81.6)$, respectively. For the non-equiatomic quinary HEAs, a special composition $(\mathrm{ABCD})_{24} \mathrm{E}_{64}$ was chosen in which an principle element $\mathrm{E}$ has highest atomic concentration of $40 \%$ (64 atoms, deviating from equiatomic composition by $20 \%$ ), and all the rest having the same lower atomic concentration of $15 \%$ (24 atoms) (Fig. 1 c, d). We intentionally used the SQS structures to represent the situation of very strong fluctuation in local atomic fraction in $\mathrm{CrFeNiCoPd}$ HEA experimentally observed by Ding et. al ${ }^{[19]}$. All SQS structures used in this work were generated by the mcsqs code implemented in the Alloy Theoretic Automated Toolkit (ATAT) package. ${ }^{[22]}$

Radial distribution functions (RDF) in Fig. 1 demonstrate how well our generated SQS structures are in representing the randomness characteristics of solid solution HEA. For the equiatomic SQS quinary HEAs, all RDF of pairs are nearly the same (Fig.1 a, b), while in the nonequiatomic $(\mathrm{ABCD})_{24} \mathrm{E}_{64} \mathrm{SQS}$ structures due to higher atomic concentration of $\mathrm{E}$, it is the highest for $\mathrm{AE}$ pair and all the other RDF pairs are smaller and very similar (Fig.1 c, d).

\subsection{Computational Methods}

All total energy calculations at $0 \mathrm{~K}$ temperature were carried out by spin-polarized DFT calculations using the projector augmented wave (PAW) method ${ }^{[26]}$ as implemented in the Vienna Ab Initio Simulation Package (VASP). ${ }^{[27-29]}$ Also the generalized gradient approximation and the Perdew-Burke-Ernzerhof pseudo potential (GGA-PBE) ${ }^{[30,31]}$ were used in treating the exchange and correlation energy. An energy cutoff wavefunction was set 
Fig. 1 Radial distribution functions of the equiatomic $\operatorname{ABCDE}(\mathrm{a})$ and (b), and nonequiatomic $(\mathrm{ABCD})_{24} \mathrm{E}_{64}$ (c) and (d) SQS structures used in this work. Insets are fcc (a) and (c), and bcc (b) and (d) SQS structures as described in the main text
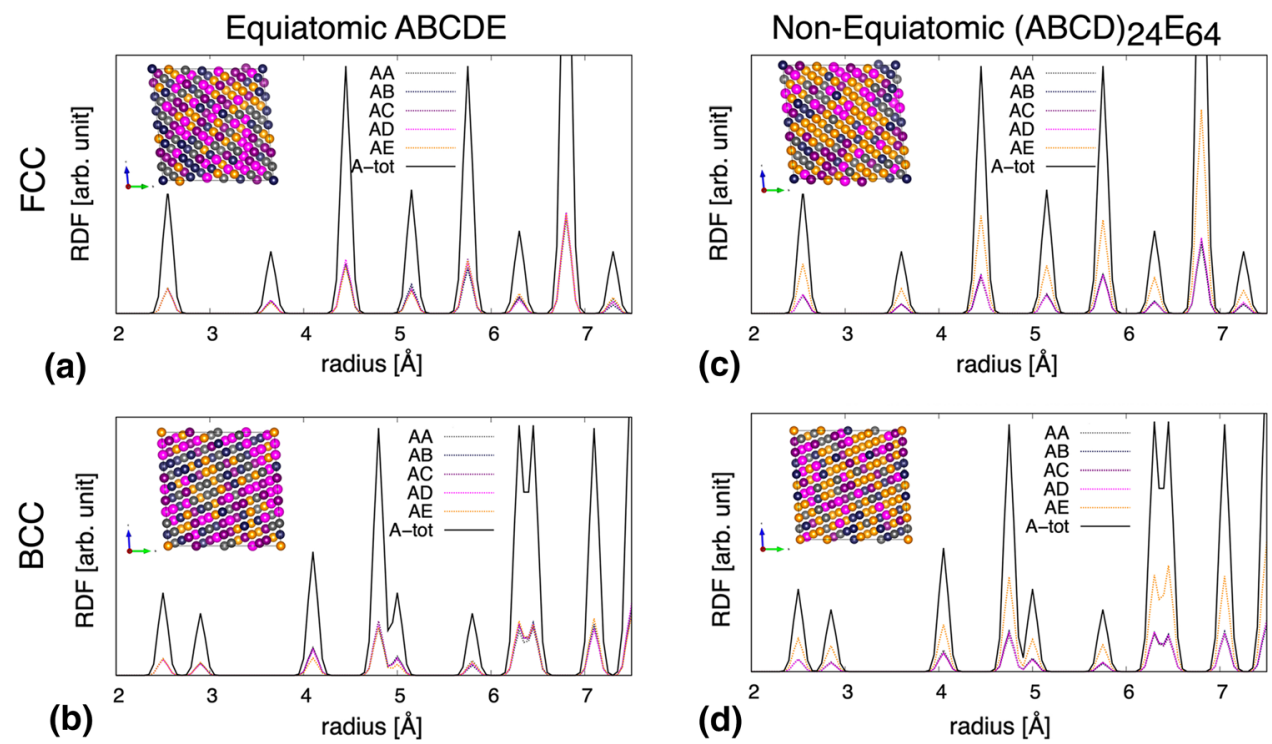

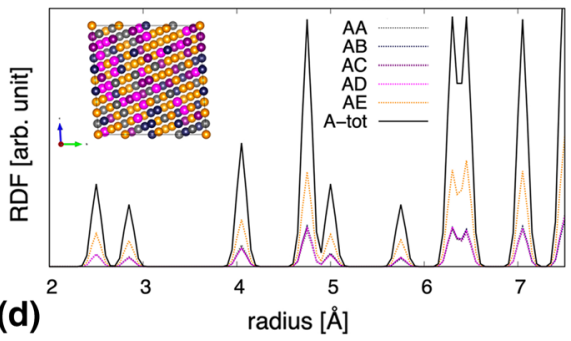

up at $400 \mathrm{eV}$ and the Monkhorst-Pack k-point mesh ${ }^{[32]}$ of $3 \times 3 \times 3$ was used in the Brillouin-zone integrations. All SQS structures were fully relaxed until all force components smaller than $2 \mathrm{meV} / \AA ̊$.

The total Gibbs free energy as a function of temperature, $\mathrm{G}_{\text {tot }}(T)$, can be calculated by summarizing the total energy of electrons $\mathrm{E}^{\mathrm{OK}}$ at $0 \mathrm{~K}$ and contributions of thermal vibration $\mathrm{F}_{\mathrm{vib}}(\mathrm{T})$, thermal electronic $\mathrm{F}_{\mathrm{el}}(\mathrm{T})$, and ideal mixing configurational entropy $\mathrm{S}_{\text {config }}$, as follow:

$\mathrm{G}_{\mathrm{tot}}(\mathrm{T})=\mathrm{E}^{0 K}+F_{\mathrm{vib}}(\mathrm{T})+\mathrm{F}_{\mathrm{el}}(\mathrm{T})-\mathrm{T} * \mathrm{~S}_{\text {config }}$

To obtain the temperature dependent thermal vibrational free energy $F_{v i b}(T)$, the quasiharmonic Debye-Grüneisen model was used as implemented in Gibbs 2 code ${ }^{[33,34]}$ with modified Poisson ratio being of 0.34 . The thermal electronic free energy $\mathrm{F}_{\mathrm{el}}(\mathrm{T})$ can be obtained using:

$\mathrm{F}_{\mathrm{el}}(\mathrm{T})=\mathrm{E}_{\mathrm{el}}-\mathrm{T} * \mathrm{~S}_{\mathrm{el}}$

where:

$E_{\mathrm{el}}=\int n(\varepsilon) f \varepsilon d \varepsilon-\int^{\varepsilon_{F}} n(\varepsilon) \varepsilon d \varepsilon$

$\mathrm{S}_{\mathrm{el}}=-\mathrm{k}_{\mathrm{B}} \int n(\varepsilon)[f \ln f+(1-f) \ln (1-f)] d \varepsilon$

In Eqs $(3,4), n$ and $f$ are electronic density of state and the Fermi distribution function, respectively, $\varepsilon_{F}$ being Fermi energy. The thermal electronic free energy was also calculated with ATAT package. ${ }^{[22]}$

Finally, the ideal configurational entropy of mixing can be expressed as:

$S_{\text {config }}=-R \sum_{i=1}^{5} c_{i} \ln c_{i}$ where $c_{i}$ is concentration of $i^{\text {th }}$ constituent element. This has been taken as a good approximation for the fully random system, and should be appropriate for multi-components HEA. Gao et. al. [43] have compared the configurational entropies for various equiatomic quaternary alloys (FCC, BCC, HCP) using DFT combining with Monte Carlo/Molecular Dynamic method and the entropy of ideal mixing and showed that the values of the ideal mixing entropy are fairly close to the values using more accurate methods.

\section{Results and Discussions}

\subsection{Structural, Electronic Structure and Magnetic Properties}

Using our generated SQS structures, two sets of quinary HEA structures have been considered by substituting dummy elements $(\mathrm{A}, \mathrm{B}, \mathrm{C}, \mathrm{D}, \mathrm{E})$ by those of interest $(\mathrm{Cr}$, $\mathrm{Fe}, \mathrm{Ni}, \mathrm{Co}, \mathrm{Mn}$, and $\mathrm{Pd}$ ): $\mathrm{Cr}-\mathrm{Fe}-\mathrm{Ni}-\mathrm{Co}-\mathrm{Mn}$, and $\mathrm{Cr}-\mathrm{Fe}-\mathrm{Ni}-$ Co-Pd. Hereafter, they are shortly abbreviated as CFNCM and CFNCP for the equiatomic quinary HEAs, respectively, while the non-equiatomic ones, such as $(\mathrm{CrFeNiCo})_{24} \mathrm{Mn}_{64}$ for example, is denoted as CFNCMMn64 and so on. In total, there are 4 equiatomic SQS structures and 20 non-equiatomic SQS that have been fully relaxed. Table 1 summarizes structural and magnetic properties of all optimized SQS structures including supercell lattice parameters, volume per atom $\mathrm{V}_{0}$, root mean square displacement (RMSD), as well as magnetic moment per atom for two systems. Details of the final relaxed SQS structures are shown in supporting information (SI) (Fig. S1). 


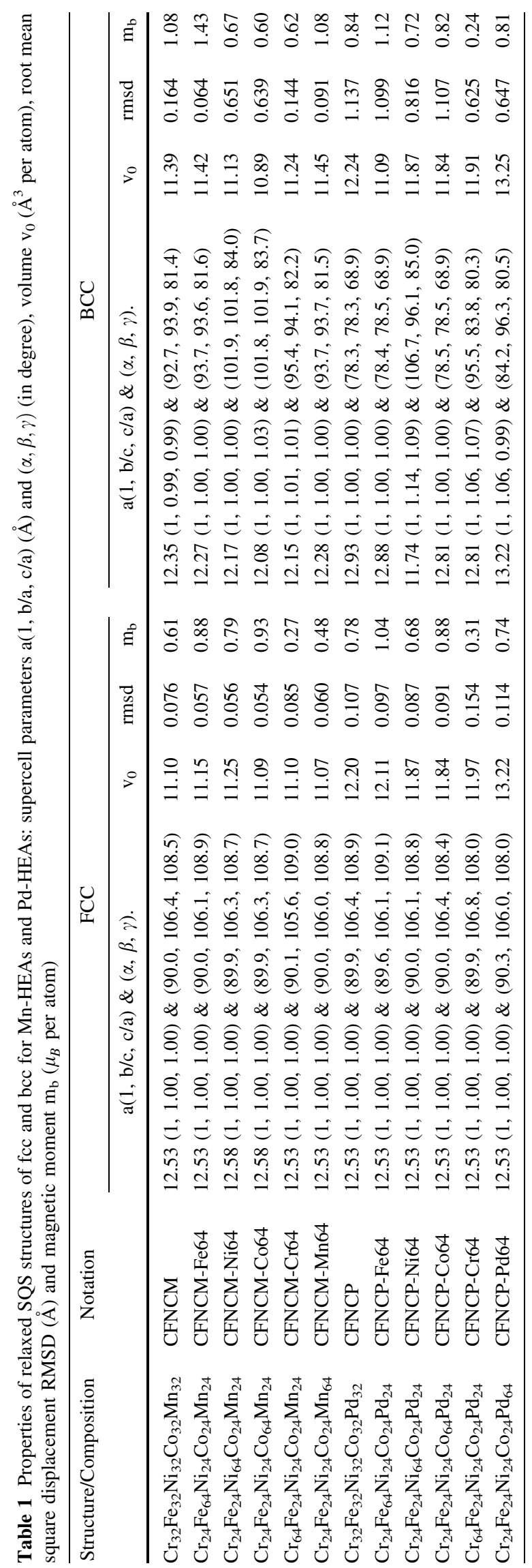


We firstly focus on structural properties of the fully relaxed fcc structures. Our DFT calculations show that there are small cell distortions and ionic local displacements during the relaxations for both Mn- and Pd-sets of fcc SQS structures. The supercell lattice parameters of all the relaxed fcc SQS structures are very similar to each other, and the deviations from the pristine cells are found to be less than $1 \%$. The calculated RMSD values of the Pdfcc set are obviously bigger than those of the Mn-set implying more lattice distortion for the Pd-HEA systems (see Fig. S1), but all of them are smaller than $0.2 \AA$. Okamoto and co-authors ${ }^{[21]}$ have reported RMSD value of $0.05 \AA$ for fcc $\mathrm{CrMnFeNiCo}$ in their 320-atoms SQS structure, which is comparable with our result $(0.076 \AA$ for CFNCM). The authors also confirmed that the difference between their theoretical and experimental results is very small, being only $0.017 \AA$. Although the values of atomic displacements in these HEAs are very small, it has been found that the mechanical properties of the HEAs relate to the averaged atomic displacement, and the yield strengths of some typical HEAs can be scaled and predicted using their RMSD values. ${ }^{[21]} \mathrm{We}$ believe that introducing the large size element Pd into the $3 \mathrm{~d}$ elements based solid solution results in the bigger average atomic displacement in Pd-HEAs, which might be the direct reason leading to its promising mechanical properties.

As demonstrated in Table 1, the difference in volume $\mathrm{v}_{0}$ among considered structures in the fcc Mn-HEA set is minuscule, despite the fact that there is drastic difference in concentration of constituent elements. The biggest volume is found to be $11.25 \AA^{3}$ for Ni-rich CFNCM-Ni64, which is only $0.18 \AA^{3}$ bigger than the smallest Mn-rich CFNCMMn64. This is due to the well-known fact that the values of the atomic radii of the Cantor elements are very close. ${ }^{[21]} \mathrm{A}$ similarity is also found in the fcc Pd-HEA set where the concentration of Pd is kept constant at $15 \%$. Substituting Mn with Pd always results in bigger volume $\mathrm{v}_{0}$ because of $\mathrm{Pd}$ having bigger atomic radius than $\mathrm{Mn}$. The calculated values of $\mathrm{v}_{0}$ for the equiatomic fcc CFNCM and CFNCP are $11.10 \AA^{3}$ and $12.20 \AA^{3}$ which can be converted straightforwardly to lattice constants of 3.54 and $3.65 \AA$, which is consistent with previous findings. ${ }^{[35,36]}$ In the fcc Pd-HEA set, it is observed that $\mathrm{v}_{0}$ decreases from 13.22 to $12.20 \AA^{3}$, and to $11.84 \AA^{3}$ as Pd concentration decreases subsequently from $40 \%$ (CFNCP-Pd64) to $20 \%$ (CFNCP), and to $15 \%$ (CFNCP-Co64) (see Table 1). This change in $\mathrm{v}_{0}$ has been also reported for other Pd concentrations $\left(\mathrm{Co}_{7} \mathrm{Cr}_{7} \mathrm{Fe}_{7} \mathrm{Ni}_{7} \mathrm{Pd}_{4}, \quad \mathrm{Co}_{6} \mathrm{Cr}_{7} \mathrm{Fe}_{7} \mathrm{Ni}_{6} \mathrm{Pd}_{6}, \quad \mathrm{Co}_{4} \mathrm{Cr}_{8} \mathrm{Fe}_{8} \mathrm{Ni}_{4} \mathrm{Pd}_{8}\right.$, $\mathrm{Co}_{5} \mathrm{Cr}_{6} \mathrm{Fe}_{6} \mathrm{Ni}_{5} \mathrm{Pd}_{10}$ ) by Calvo-Dahlborg et. al ${ }^{[35]}$ using smaller fcc SQS structure of 32-atoms, where similar trend was found.
Our DFT calculations revealed a strong substitutional effect of Pd with $\mathrm{Mn}$ in simple bcc phase. Being very different from fcc structures, we could not stabilize any Pdcontaining bcc structures considered in this study even for systems containing high concentration of bcc-favoring components like $\mathrm{Fe}$ and $\mathrm{Cr}$ (CFNCP-Fe64, CFNCP-Cr64). The Pd- bcc set undergoes severe cell shape and lattice distortion during structural optimization. For instance, the angles between principal axes in the equiatomic CFNCP are $(78.3,78.3,68.9)$ compared to the starting ones (93.8, 93.8, 81.6). The severe local lattice distortion can be pronouncedly seen in the non-equiatomic CFNCP-Ni64, CFNCP-Cr64, and CFNCP-Pd64 (see Fig. S1 i, k, 1) where layers of atoms are regrouped or irregularly redistributed. As shown in Table 1, the calculated RMSD for the final bcc Pd-HEA structures are all greater than $0.62 \AA$ and can reach up to $1.14 \AA$ (CFNCP). The large RMSD values are consequence of distortion of both cell shape and lattice. Although the larger RMSD due to introducing inhomogeneous feature leads to better mechanical properties, it is also important to consider the trade-off between inhomogeneous and stability.

For the bcc set with Mn, similar phenomenon was found for the non-equiatomic bcc CFNCM-Ni64 and CFNCMCo64 (see Table 1 and Fig. S1 c, d) where high concentrations in $\mathrm{Ni}$ and Co components are favoring fcc lattice rather than bcc. The other Mn-HEA compositions, equiatomic and non-equiatomic, can stabilize in bcc lattice and show a much smaller cell shape and ionic relaxations. Li et. al ${ }^{[37]}$ had experimentally synthesized $\mathrm{FeCoNi}(\mathrm{MnAl}) \mathrm{x}$ HEAs and reported a Al-concentration dependence for formation of single fcc phase $(0 \leq \mathrm{x} \leq 0.5)$, a mixture of fcc and bcc structures $(0.5 \leq \mathrm{x} \leq 1.5)$, and bcc phase ( $\mathrm{x}$ $\geq 2.0$ ). Other DFT calculations carried out by Feng et. al ${ }^{[38]}$ have confirmed this observation. Also in Ref. ${ }^{[39]}$ the authors using DFT-CPA approach concluded that adding Al to $\mathrm{CrMnFeCoNi} \mathrm{HEA} \mathrm{can} \mathrm{trigger} \mathrm{the} \mathrm{phase} \mathrm{transfor-}$ mation from fcc to bcc. Therefore, we suggest that in order to stabilize quinary Pd-HEAs and other non-equiatomic Mn-HEAs in simple bcc phase, one needs to add certain amount of bcc stabilizer such as Al.

The radial distribution functions (RDFs) plotted in Fig. 2 and Fig. S2 further support our above conclusion that the Pd-containing HEAs and CFNCM-Ni64, CFNCM-Co64 considered in this study could not stabilize in bcc solid solution phase. RDFs of those undergoing great cell shape and lattice distortion completely lost the standardized RDF characteristics of bcc lattice (see Fig. $1 \mathrm{~b}, \mathrm{~d}$ as reference). Their peaks in RDF spectra are found relocating at positions that quite similar with those of fully relaxed corresponding fcc structures. More surprisingly, RDFs of $b c c$ CFNCP, CFNCP-Fe64, and CFNCP-Co64 resemble exactly the same as for corresponding fcc ones (Fig. 2 b, 


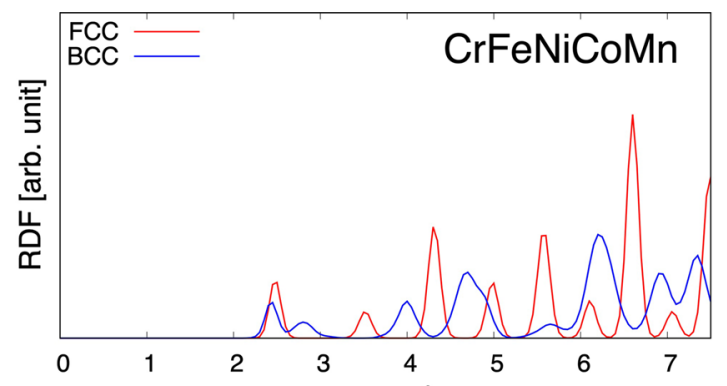

(a)

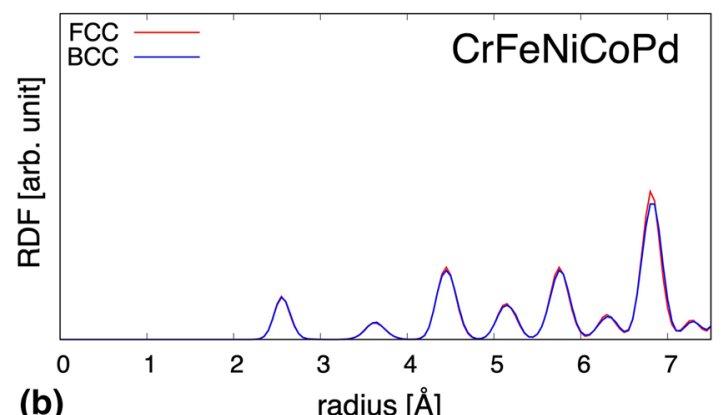

(b)
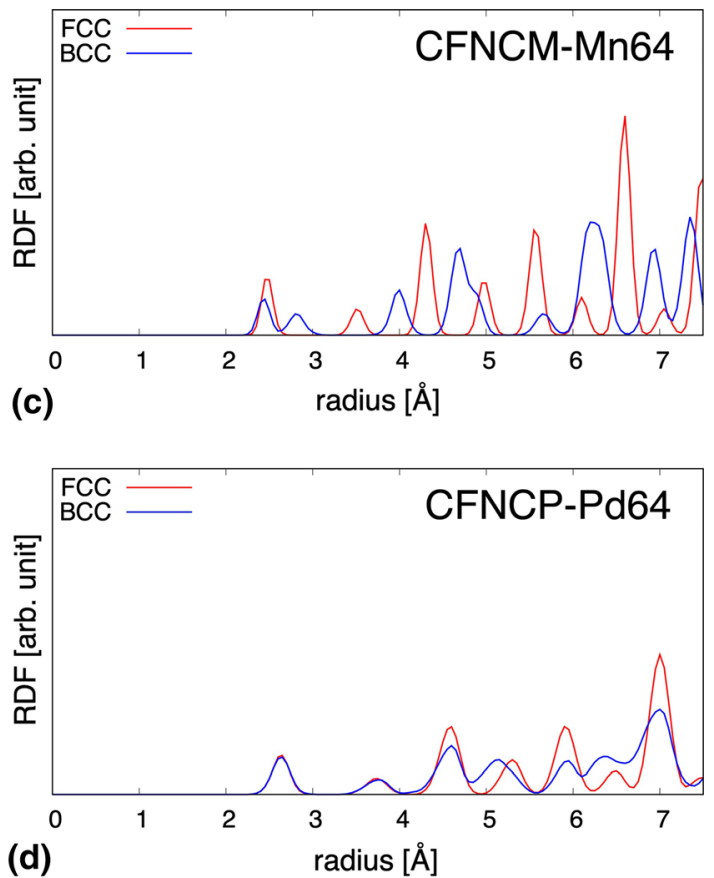

Fig. 2 Radial distribution function of the fully relaxed equiatomic CFNCM (a), CFNCP (b); and non-equiatomic CFNCM-Mn64 (c), CFNCPPd64 (d) SQS structures

Fig. S2 e, g). For the fcc Mn- and Pd- sets, in contrast, their RDFs show a lot of similarities with the perfect fcc lattice, excepted for the width of peaks. It is easily seen that all peaks in the fcc sets are broaden due to ionic relaxation. The RDF peaks for the Pd-HEAs are shifted to bigger radius compared to Mn-ones, and their widths are found to be also wider. The findings are in good agreement with other studies on equiatomic HEAs FeCoNiCrMn, FeCo$\mathrm{NiCrPd}$, and binary $\mathrm{Ni}_{80} \mathrm{Pd}_{20}$ alloy. ${ }^{[40,}{ }^{41]}$ In addition, the authors have clearly demonstrated that RDF width broadening is because of the fluctuation in nearest-neighbor distances between metal atoms.

Figure 3 shows the renormalized total density of state (DOS) (per atom) and the most important d-orbital partial DOS of the equiatomic CFNCM and non-equiatomic CFNCM-Mn64 HEAs in comparison with the corresponding Pd-ones. All the rest can be found in Fig. S3. Despite of the difference in the (P)DOS shapes from system to system, we recognized some common features between them. Firstly, all shows the majority in spin-up PDOS for ferromagnetic elements $\mathrm{Fe}, \mathrm{Ni}, \mathrm{Co}$, and $\mathrm{Pd}$ (fcc) in which their PDOS shapes could be different from those of corresponding pure elements. Pure fcc $\mathrm{Mn}$ is antiferromagnetic however in all considered fcc HEA systems the majority shows it in spin-up states as well. Other antiferromagnetic element $\mathrm{Cr}$, on the orther hand, has majority in spin-down PDOS. Its local spin state is most likely preferred in opposite direction when surrounded by other ferromagnetic elements, and magnetic moment of HEAs will be reduced as consequence. Secondly, replacing Mn with Pd tends to enhance the asymmetrical characteristics between up- and down- PDOS. Therefore, the magnetic property of the HEAs containing Pd can be improved, for instance, magnetic moment per atom of Pd-rich fcc CFNCP-Pd64 is about 1.5 times bigger than Mn-rich fcc CFNCM-Mn64 (see Table 1). Finally, we observed that in the stable Cr-rich fcc CFNCM-Cr64 and CFNCP-Cr64 systems the asymmetrical characteristics between up- and down- PDOS are reduced for $\mathrm{Fe}, \mathrm{Ni}, \mathrm{Co}, \mathrm{Mn}$ and Pd (see Fig. S2 d, h) implying the reduction in magnetic moment of those constituent elements. Indeed, the magnetic moments of the fcc CFNCM-Cr64 and CFNCP-Cr64 systems are reported to be the lowest among our considered systems, being 0.27 and $0.31 \mu_{B}$, respectively (see Table 1). Above analysis suggests the possibility of tailoring the magnetic properties of HEAs using specific combination of composition elements.

\subsection{Thermodynamics and Energetics}

We furthermore investigated the thermodynamics and energetics of Mn- and Pd-HEAs. As already discussed in the previous section, since all Pd-HEAs in bcc structures are unstable due to a large structural distortion; following discussion focuses on the fcc phases of $\mathrm{Mn}-$ and $\mathrm{Pd}-$ quinary HEAs only.

Displayed in Fig. 4 is the comparison for Mn-HEAs and Pd-HEAs of various finite temperature effects contributing 
Fig. 3 Total and d-orbital partial density of states of the fully relaxed equiatomic CFNCM (a), CFNCP (b); and non-equiatomic CFNCM-Mn64 (c), CFNCP-Pd64 (d) SQS structures

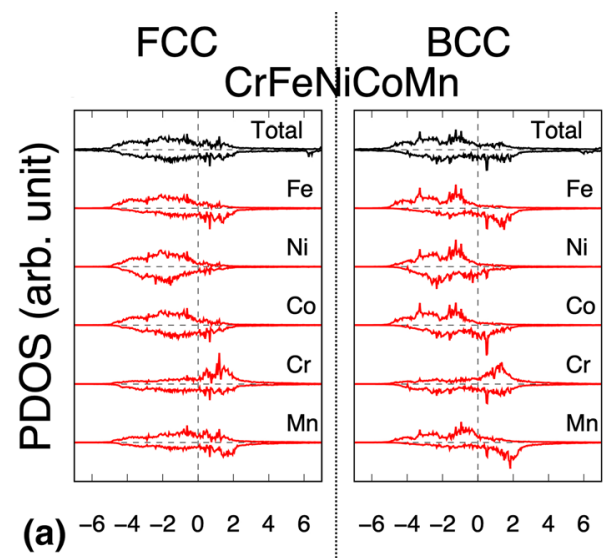

CrFeNiCoPd

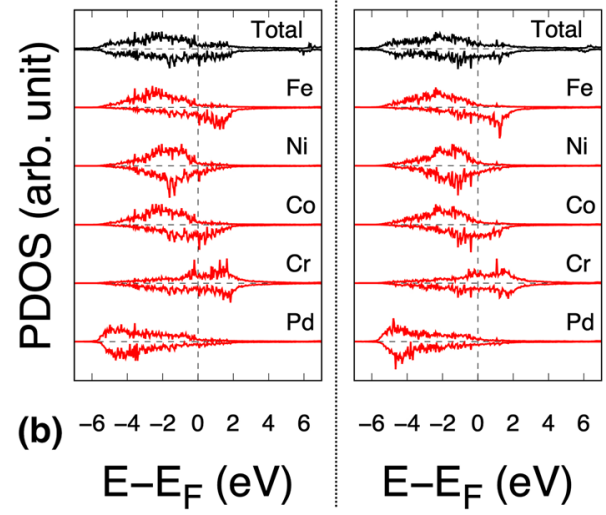

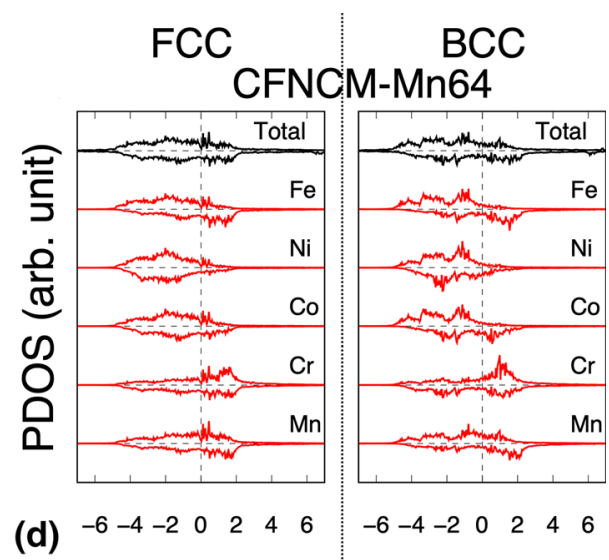

CFNCP-Pd64

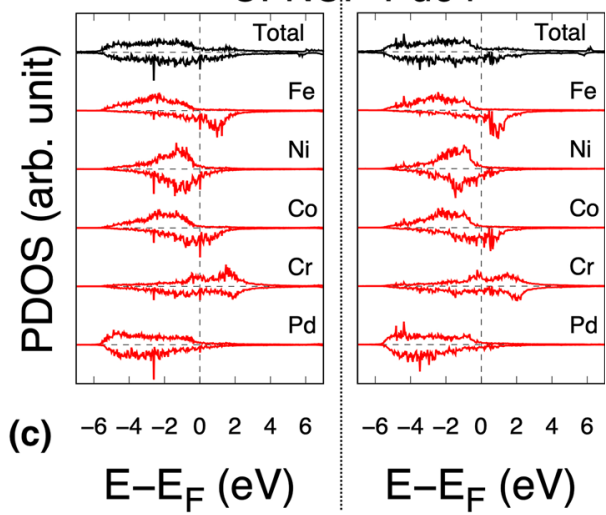

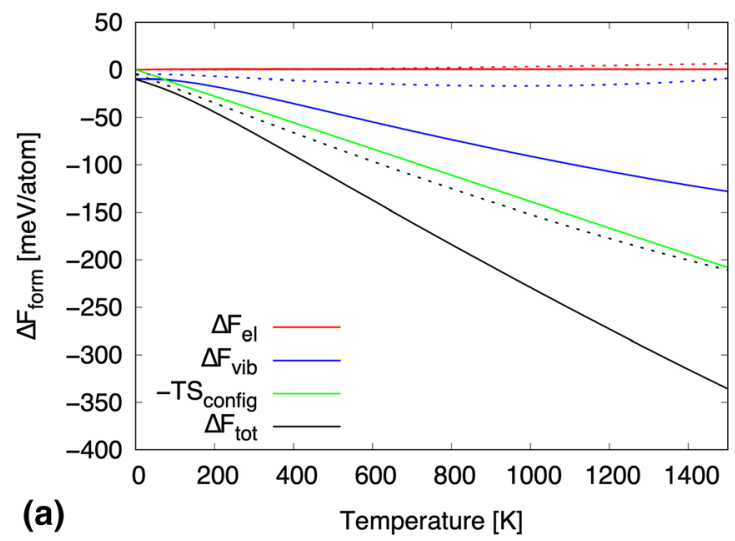

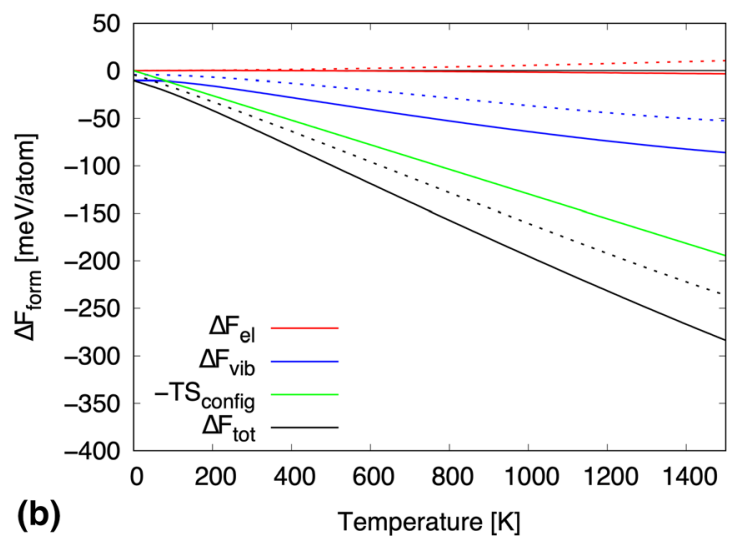

Fig. 4 Thermodynamic contributions to Gibbs formation free energies in the fully relaxed equiatomic CFNCM, CFNCP (a); and non-equiatomic CFNCM-Mn64, CFNCP-Pd64 (b) fcc SQS structures. Results for the fcc Mn-HEAs are in solid lines, and for the fcc Pd-HEAs are in dashed lines

to the Gibbs formation energy up to $1400 \mathrm{~K}$, including the lattice vibration $\Delta F_{v i b}$, thermal electronics $\Delta F_{e l}$, and ideal mixing configurational free energy $-T S_{\text {config, }}$ in the equiatomic CFNCM, CFNCP and non-equiatomic Mn-rich CFNCM-Mn64, Pd-rich CFNCP-Pd64 HEAs. The contributions of vibrational and thermal electronic free energies are shown separately for all fcc $\mathrm{Mn}$ - and Pd- structures in Figs. S4 and S5. It is easily seen that the ideal configurational entropy term is the biggest contribution to free energies in both equiatomic and non-equiatomic systems. Since our specific setting of non-equiatomic $(\mathrm{ABCD})_{24} \mathrm{E}_{64}$ SQS structure has 4 constituent elements with equal concentration $(15 \%)$, it still maintains high-entropy effect. Indeed, at $\mathrm{T}=1000 \mathrm{~K}$, the calculated configurational free energies $-\mathrm{TS}_{\text {config }}$ for the equiatomic CFNCM/P and nonequiatomic CFNCM/P-X64 are very close, e.g -139 and $130 \mathrm{meV} /$ atom, respectively. Fig. 4 shows that the lattice vibrational contribution has stronger effect of stabilization in the relevant Mn-HEAs compared to the Pd- counterparts. 
In particular, the contribution of $\Delta F_{v i b}$ is quite small in the fcc CFNCP and partially reduced by $\Delta F_{e l}$, making the total contribution $\Delta F_{\text {tot }}$ very close to the configurational con-

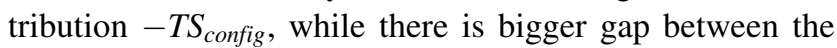
two in the fcc CFNCM.

Combining all the finite temperature contributions allows us to visualize the Gibbs formation energy with increasing temperature as shown in Fig. 5. For each 2D plots in Fig. 5, the center circles represent for the equiatomic CFNCM and CFNCP HEAs, and the circles at the $\% \mathrm{X}$-axis, \%Ni-axis in Fig. 5a for instance, represents for the Ni-rich non-equiatomic fcc CFNCM-Ni64 HEA. At OK all the studied fcc HEAs have positive formation energies, reflecting the fact that they are not the ground states but the metastable ones. The computed values for the equiatomic CFNCM and CFNCP are very close to each other, 79 and $81 \mathrm{meV} /$ atom, respectively, which are comparable with previously calculated values. ${ }^{[6,35,42]}$ Among our calculated structures, the fcc Ni-rich HEAs (CFNCM-Ni64, CFNCP-Ni64) and Pd-rich (CFNCP-Pd64) are found to be the relatively stable structures with the formation energies of being lower than $50 \mathrm{meV} / \mathrm{atom}$. On the other hand, increasing the concentration of antiferromagnetic $\mathrm{Mn}$ and $\mathrm{Cr}$ elements is energetically unfavorable. The highest formation energy, $132 \mathrm{meV} / \mathrm{atom}$, is found for the fcc CFNCP-Cr64.

When the temperature stabilization effect of configurational entropy and lattice vibration are considered, the Gibbs formation energies $G_{f}$ of the fcc quinary Mn- and Pd-HEAs are decreased and change to negative. Indeed, at $\mathrm{T}=600 \mathrm{~K}$ almost all the fcc quinary Mn- and Pd-HEAs became stable, except for the Cr-rich CFNCM-Cr64 and CFNCP-Cr64 (see Fig. 5). The largest reduction in Gibbs

Fig. 5 Gibbs formation free energies at finite temperature of fcc Mn-HEAs (a) and Pd-HEAs (b). The radius of each circles is proportional to the absolute value of the formation energy
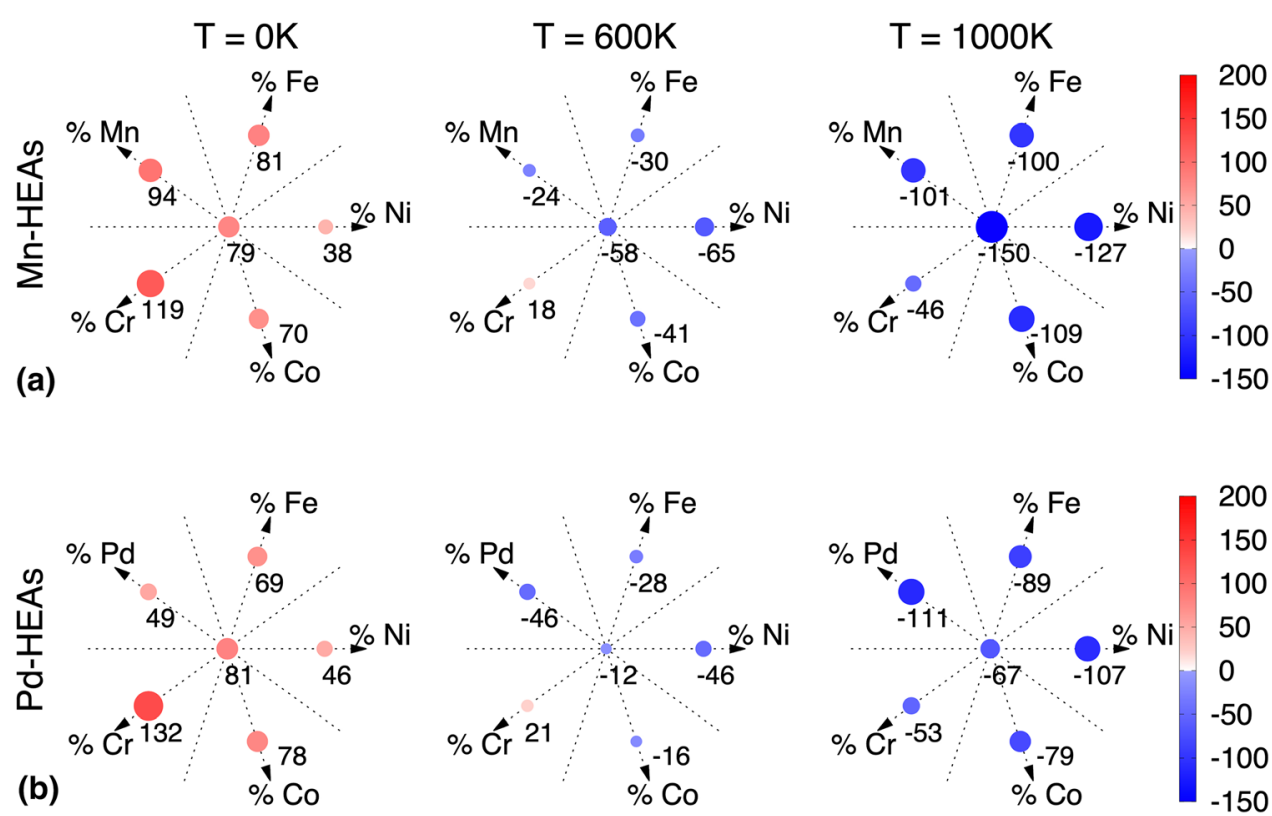

formation energy, $\Delta G_{f}=-137 \mathrm{meV} / \mathrm{atom}$, was found for the equiatiomic CFNCM. At $\mathrm{T}=1000 \mathrm{~K}, G_{f}$ values of all computed systems are negative and the fcc Mn-HEAs are more stable compared to the corresponding Pd-ones. The fcc equiatomic CFNCM is the most stable structure, $G_{f}=$ $-150 \mathrm{meV} / \mathrm{atom}$, but when substituting $\mathrm{Mn}$ by $\mathrm{Pd}$, the CFNCP HEA becomes one of the least stable ones. This big difference could be explained by comparing the contributions of vibrational and thermal electronic free energies for all the studied systems, see Figs. S4, S5, since the configurational mixing entropies are almost the same. Concerning the thermal electronic contribution $\Delta F_{e l}$ alone, it shows the destabilization effect for all fcc Pd-HEAs and for some fcc Mn-HEAs (CFNCM, CFNCM-Ni64, CFNCM-Co64), but in overall $\left|\Delta F_{e l}\right|$ is less than $15 \mathrm{meV} /$ atom over the whole range of considered temperature for all cases (see Fig. S4 b, S5 b) and can be neglected. The stabilization effect of vibrational free energy, on the other hand, is much more significant for all cases. The effect is maximized in the case of fcc equiatomic CFNCM. When Pd substituting Mn, $\Delta F_{v i b}$ of the fcc CFNCP is always of the highest value for $\mathrm{T}>600 \mathrm{~K}$, therefore cannot stabilize the CFNCP as much as for other HEAs.

In Fig.5, it is interesting to notice that the trends in the variation of the formation free energies with increasing temperature in Mn-HEAs and Pd-HEAs, respectively, and compare the two systems. In Mn-HEAs, the formation free energy of the equiatomic one (central circle) is getting more negative than the non-equiatomic ones (X\%-axis), indicating that at finite temperature, the equiatomic $\mathrm{Mn}$ HEA is more preferred than non-equiatomic ones. Whereas, the situation in Pd-HEAs is opposite. While the temperature is increasing, the value for central circle is

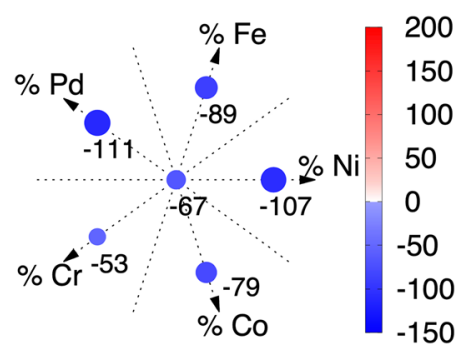


obviously smaller than those for all $\mathrm{X} \%$-axes, showing that in Pd-HEAs, various non-equiatomic systems tend to more stable than the equiatomic one. Such observation is consistent with the Ding et al.'s report ${ }^{[19]}$, i.e., the existing big composition fluctuations in $\mathrm{FeCoNiCrPd}$.

We are wondering what happens in Pd-HEAs and explored this phenomenon through following analysis using a hypothesis model for quinary HEAs where both equiatomic and non-equiatomic systems co-exist. Here we imagine a sample of CFNCY ( $\mathrm{Y}=\mathrm{Mn}, \mathrm{Pd})$ equal in constituent concentrations but consisting of 5 types of the nonequiatomic CFNCY-X64 in the same portion distributed randomly in the sample. So, the hypothesized CFNCY sample is equiatomic as average but inhomogeneous in local environment, which is taken as the model of hypothetical equiatomic quinary HEAs. For simplicity, the formation energy of the hypothetical equiatomic system is approximately equal to the average of the formation energies of all 5 types CFNCY-X64. Figure 6 shows the estimated Gibbs formation energies of both the hypothesized and of the real modeled equiatomic quinary fcc CFNCM and CFNCP as a function of temperature. It is obvious that for CFNCM, the real equiatomic fcc CFNCM has lower formation energy than the hypothesized one, such energetic preference implies that when one tries to fabricate a quinary CFNCM sample starting with equal atomic concentration of constituents, it is more likely that the equiatomic CFNCM HEA will form with homogeneous elements distribution. This is different for CFNCP, where the hypothesized equiatomic model is more stable than the real one, which leads to a high possibility that equiatomic CFNCP will form with inhomogeneous element distribution, even with a strong fluctuation in local atomic fraction.

This assumption is confirmed by the experimental results reported by Ding et.al. ${ }^{[19]}$. Using energy-dispersive $\mathrm{X}$-ray spectroscopy the authors had investigated the atomic-scale element distribution in equiatomic fcc

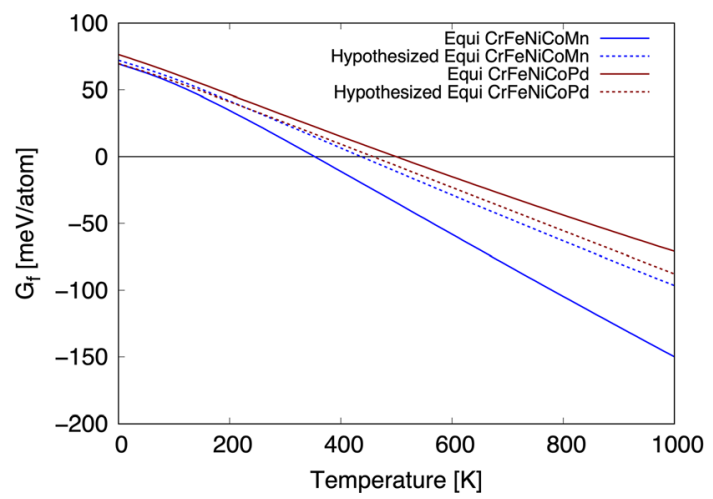

Fig. 6 Gibbs formation free energies as a function of temperature for the hypothesized (dashed lines) and real modeled (solid lines) equiatomic fcc CFNCM (blue) and CFNCP (dark-brown)
CrFeNiCoMn Cantor alloy, and also compared with fcc $\mathrm{CrFeNiCoPd} \mathrm{HEA}$. For the $\mathrm{CrFeNiCoMn}$ sample, they found a small fluctuation in local atomic fraction around the equiatomic line for all five elements. In contrast to the Cantor alloy, the atomic fraction lines of all five elements in $\mathrm{CrFeNiCoPd}$ alloy show a much wider fluctuation with the maxima reaching up to $58 \%$. The fluctuations in both $\mathrm{CrFeNiCoMn}$ and $\mathrm{CrFeNiCoPd}$ samples seem to be random.

\section{Conclusion}

In this work, we carried out comprehensive DFT calculations combined with the quasiharmonic Debye-Grüuneisen approximation for both equiatomic and non-equiatomic compositions of $\mathrm{CrFeNiCoMn}$ Cantor and $\mathrm{CrFeNiCoPd}$ quinary HEAs based on SQS models. Similarities and differences between the two systems as consequences of Pd-to-Mn substitution effect have been highlighted in terms of structural, electronic structure, and magnetic properties, as well as thermodynamics and energetics. It has been found that substituting Mn with Pd in Cantor alloy introduced a inhomogeneous feature into the HEA, which increases the average atomic local displacement of the fcc $\mathrm{CrFeNiCoPd}$ to a moderate amount, consequently enhances the mechanical properties of the new HEA. It is also revealed that the stability of equiatomic/near-equiatomic composition is preferable than the extreme non-equiatomic in fcc $\mathrm{CrFeNiCoMn} \mathrm{HEAs,} \mathrm{whereas} \mathrm{the} \mathrm{situation} \mathrm{in} \mathrm{fcc}$ $\mathrm{CrFeNiCoPd}$ is reverted, that means the mixture of various non-equiatomic compositions is more preferred due to the local inhomogeneous feature, especially for Pd-rich and Ni-rich $\mathrm{CrFeNiCoPd}$ HEAs. These findings can explain some experimental observations reported recently. $\mathrm{CrFe}-$ $\mathrm{NiCoMn}$ and $\mathrm{CrFeNiCoPd}$ HEAs are among interesting multi-principal elements alloys representative for novel physical properties and with the potential for advanced applications; and more studies are needed to fully understand the systems. Our work is an effort to enrich the thermodynamic databases of $\mathrm{CrFeNiCo}(\mathrm{Mn} / \mathrm{Pd}) \mathrm{HEAs}$ and could be useful for guiding material design and engineering, as well as for other future works.

Supplementary InformationThe online version contains supplementary material available at https://doi.org/10.1007/s11669021-00900-1.

Acknowledgments The authors acknowledge the Center for Computational Materials Science of the Institute for Materials Research, Tohoku University, for the support of the supercomputing facilities. 


\section{References}

1. B. Cantor, I.T.H. Chang, P. Knight, and A.J.B. Vincent, Microstructural Development in Equiatomic Multicomponent Alloys, Mater. Sci. Eng. A, 2004, 375-377, p 213-218.

2. P.K. Huang, J.W. Yeh, T.T. Shun, and S.K. Chen, Multi-principal-element Alloys with Improved Oxidation and Wear Resistance for Thermal Spray Coating, Adv. Eng. Mater., 2004, 6, p 74-78.

3. J.W. Yeh, S.K. Chen, S.J. Lin, J.Y. Gan, T.S. Chin, T.T. Shun, C.H. Tsau, and S.Y. Chang, Nanostructured High-Entropy Alloys with Multiple Principal Elements: Novel Alloy Design Concepts and Outcomes, Adv. Eng. Mater., 2004, 6, p 299-303.

4. D.B. Miracle, and O.N. Senkov, A Critical Review of High Entropy Alloys and Related Concepts, Acta Mater., 2017, 122, p 448-511.

5. Y. Ikeda, B. Grabowski, and F. Kormann, Ab Initio Stabilities and Mechanical Properties of Multicomponent Alloys: A Comprehensive Review for High Entropy Alloys and Compositionally Complex Alloys, Mater. Charact., 2019, 147, p 464-511.

6. M.C. Gao, J.W. Yeh, P.K. Liaw, and Y. Zhang, High-Entropy Alloys: Fundamentals and Applications. Spinger, New York, 2016.

7. J. Kitagawa, S. Hamamoto, and N. Ishizu, Cutting Edge of HighEntropy Alloy Superconductors from the Perspective of Materials Research, Metals, 2020, 10, p 1708.

8. H. Peng, Y. Xie, Z. Xie, Y. Wu, W. Zhu, S. Liang, and L. Wang, Large-Scale and Facile Synthesis of a Porous High-Entropy Alloy $\mathrm{CrMnFeCoNi}$ as an Efficient Catalyst, J. Mater. Chem. A, 2020, 8, p 18318-18326.

9. B. Schuh, F.M. Martin, B. Volker, E.P. George, H. Clemens, R. Pippan, and A. Hohenwarter, Mechanical Properties Microstructure and Thermal Stability of a Nanocrystalline CoCrFeMnNi High-Entropy Alloy after Severe Plastic Deformation, Acta Mater., 2015, 96, p 258-268.

10. E.J. Pickering, R. Munoz-Moreno, H.J. Stone, and N.G. Jones, Precipitation in the Equiatomic High-Entropy Alloy CrMnFeCoNi, Scr. Mater., 2016, 113, p 106-109.

11. G. Laplanche, A. Kostka, O. Horst, G. Eggeler, and E. George, Microstructure Evolution and Critical Stress for Twinning in the CrMnCoNi High-Entropy Alloy, Acta Mater., 2016, 118, p $152-163$.

12. F. Otto, A. Dlouhy, C. Somsen, H. Bei, G. Eggeler, and E. George, The Influences of Temperature and Microstructure on the Tensile Properties of a CoCrFeMnNi High-Entropy Alloy, Acta Mater., 2013, 61, p 5743-5755.

13. S.F. Liu, Y. Wu, H.T. Wang, J.Y. He, J.B. Liu, C.X. Chen, X.J. Liu, H. Wang, and Z.P. Lu, Stacking Fault Energy of FaceCentered-Cubic High Entropy Alloys, Intermetallics, 2018, 93, p 269-273.

14. Z. Li, F. Kormann, B. Grabowski, J. Neugebauer, and D. Raabe, Ab Initio Assisted Design of Quinary Dual-Phase High-Entropy Alloys with Transformation-Induced Plasticity, Acta Mater., 2017, 136, p 262-270.

15. H. Luo, Z. Li, and D. Raabe, Hydrogen Enhances Strength and Ductility of an Equiatomic High-Entropy Alloy, Sci. Reports, 2017, 7, p 9892.

16. Z. Li, Interstitial Equiatomic CoCrFeMnNi High-Entropy Alloys: Carbon Content, Microstructure, and Compositional Homogeneity Effects on Deformation Behavior, Acta Mater., 2019, 164, p 400-412.

17. W. Chen, and L. Zhang, High-Throughput Determination of Interdiffusion Coefficients for Co-Cr-Fe-Mn-Ni High-Entropy Alloys, J. Phase Equilib. Diffus., 2017, 38, p 457-465.
18. V. Verma, A. Tripathi, and K.N. Kulkarni, On Interdiffusion in FeNiCoCrMn High Entropy Alloy, J. Phase Equilib. Diffus., 2017, 38, p 445-456.

19. Q. Ding, Y. Zhang, X. Chen, X. Fu, D. Chen, S. Chen, L. Gu, F. Wei, H. Bei, Y. Gao, M. Wen, J. Li, Z. Zhang, T. Zhu, R.O. Ritchie, and Q. Yu, Tuning Element Distribution, Structure and Properties by Composition in Hing-Entropy Alloys, Nature, 2019, 574, p 223-227.

20. B. Yin, and W.A. Curtin, Origin of High Strength in the CoCrFeNiPd High-Entropy Alloy, Mater. Res. Lett., 2020, 8, p 209-215.

21. N.L. Okamoto, K. Yuge, K. Tanaka, H. Inui, and E.P. George, Atomic Displacement in the CrMnFeCoNi High-Entropy Alloy A Scaling Factor to Predict Solid Solution Strengthening, AIP $A d v ., 2016,6, \mathrm{p} 125008$.

22. A. van de Walle, P. Tiwary, M. De Jong, D.L. Olmsted, M. Asta, A. Dick, D. Shin, Y. Wang, L.-Q. Chen, and Z.-K. Liu, Efficient Stochastic Generation of Special Quasirandom Structures, Calphad, 2013, 42, p 13-18.

23. A. Zunger, S.-H. Wei, L.G. Ferreira, and J.E. Bernard, Special Quasirandom Structures, Phys. Rev. Lett., 1990, 65(3), p 353-356.

24. A.J. Zaddach, C. Niu, C.C. Koch, and D.L. Irving, Mechanical Properties and Stacking Fault Energies of NiFeCrCoMn HighEntropy Alloy, JOM, 2013, 65, p 1780-1789.

25. M.C. Gao, C. Niu, C. Jiang, and D.L. Irving, Applications of Special Quasi-Random Structures to High-Entropy Alloys Springer, High-Entropy Alloys, 2016, p 333-368

26. G. Kresse, and D. Joubert, From Ultrasoft Pseudopotentials to the Projector Augmented- Wave Method, Phys. Rev. B, 1999, 59(3), p 1758-1775.

27. G. Kresse, and J. Hafner, Ab Initio Molecular-Dynamics Simulation of the Liquid-Metal- Amorphous-Semiconductor Transition in Germanium, Phys. Rev. B, 1994, 49(20), p 14251-14269.

28. G. Kresse, and J. Furthmüller, Efficiency of Ab-Initio Total Energy Calculations for Metals and Semiconductors using a Plane-Wave Basis Set, Comput. Mater. Sci., 1996, 6(1), p 15-50.

29. G. Kresse, and J. Furthmüller, Efficient Iterative Schemes for $\mathrm{Ab}$ Initio Total-Energy Calculations using a Plane-Wave Basis Set, Phys. Rev. B, 1996, 54(16), p 11169-11186.

30. J.P. Perdew, K. Burke, and M. Ernzerhof, Generalized Gradient Approximation Made Simple, Phys. Rev. Lett., 1996, 77(18), p 3865.

31. J.P. Perdew, K. Burke, and M. Ernzerhof, Generalized Gradient Approximation Made Simple- ERRATA, Phys. Rev. Lett., 1996, 78(7), p 1396.

32. H.J. Monkhorst, and J.D. Pack, Special Points for Brillouin-Zone Integrations, Phys. Rev. B, 1976, 13(12), p 5188-5192.

33. A. Otero-de-la-Roza, and V. Luaña, Gibbs2: A New Version of the Quasi-Harmonic Model I Robust Treatment of the Static Data, Comput. Phys. Commun., 2011, 182, p 1708-1720.

34. A. Otero-de-la-Roza, and V. Luaña, Gibbs2: A New Version of the Quasi-Harmonic Model II Implementation and Test Cases, Comput. Phys. Commun., 2011, 182, p 2232-2248.

35. M. Calvo-Dahlborg, J. Cornide, J. Tobola, D. Nguyen-Manh, J.S. Wróbel, J. Juraszek, S. Jouen, and U. Dahlborg, Interplay of Electronic, Structural and Magnetic Properties as the Driving Feature of High-Entropy CoCrFeNiPd Alloys, J. Phys. D: Appl. Phys., 2017, 50, p 185002.

36. D. Ma, B. Grabowski, F. Körmann, J. Neugebauer, and D. Raabe, $\mathrm{Ab}$ Initio Thermodynamics of the CoCrFeMnNi High Entropy Alloy: Importance of Entropy Contributions beyond the Configurational One, Acta Mat., 2015, 100, p 90-97.

37. P. Li, A. Wang, and C.T. Lui, Composition Dependence of Structure, Physical and Mechanical Properties of FeCoNi(MnAl $)_{x}$ High Entropy Alloys, Intermetallics, 2017, 87, p 21-26. 
38. W. Feng, Y. Qi, and S. Wang, Effects of Mn and Al Addition on Structural and Magnetic Properties of FeCoNi-Based High Entropy Alloys, Mater. Res. Express, 2018, 5, p 106511.

39. X. Sun, H. Zhang, S. Lu, X. Ding, Y. Wang, and L. Vitos, Phase Selection Rule for Al-Doped CrMnFeCoNi High-Entropy Alloys from First-Principles, Acta Mater., 2017, 140, p 366-374.

40. Y. Tong, G. Velisa, S. Zhao, W. Guo, T. Yang, K. Jin, C. Lu, H. Bei, J.Y.P. Ko, D.C. Pagan, Y. Zhang, L. Wang, and F.X. Zhang, Evolution of Local Lattice Distortion under Irradiation in Medium- and High-Entropy Alloys, Materialia, 2018, 2, p 73-81.

41. Y. Tong, S. Zhao, K. Jin, H. Bei, J.Y.P. Ko, Y. Zhang, and F.X. Zhang, A Comparision Study of Local Lattice Distortion in Ni80Pd20 Binary Alloy and FeCoNiCrPd High-Entropy Alloy, Scripta Mater., 2018, 156, p 14-18.
42. Z. Wu, M.C. Troparevsky, Y.F. Gao, J.R. Morris, G.M. Stocks, and H. Bei, Phase Stability, Physical Properties and Strengthening Mechanisms of Concentrated Solid Solution Alloys, Curr. Opin. Solid State Mater. Sci., 2017, 21, p 221-237.

43. M.C. Gao, C. Zhang, P. Gao, F. Zhang, L.G. Ouyang, M. Widom, and J.A. Hawk, Thermodynamics of Concentrated Solid Solution Alloys, Curr. Opin. Solid State Mater. Sci., 2017, 21, p 238-251.

Publisher's Note Springer Nature remains neutral with regard to jurisdictional claims in published maps and institutional affiliations. 